\title{
Potential Distribution Measurement of Surface Discharge in $\mathrm{SF}_{6}$
}

\author{
Member Akiko KUMADA (The University of Tokyo) \\ Member Masakuni CHIBA (The University of Tokyo) \\ Member Kunihiko HIDAKA (The University of Tokyo)
}

\begin{abstract}
Sensing techniques with a Pockels crystal have been applied to directly measure the potential and electric field distribution of surface discharge creeping on a dielectric material. The transient change of potential distribution with a surface discharge, which propagates on a $780 \mu \mathrm{m}$ thickness BGO crystal in $\mathrm{SF}_{6}$ at $160 \sim$ 760 Torr pressure, is measured with high time and spatio resolution by using Pockels sensor, a CCD camera and a streak camera.

Little significant difference is recognized between the potential distribution along a negative discharge and that along a positive one. The distribution of $E_{x}$ along a streamer has a triangular profile and $E_{x}$ has its maximum near the head of it. The value of $E_{x}$ around the streamer head keeps around the critical value for the gas ionization. During subsequent stepwise propagation of the streamer, a leader, whose electrical conductivity is relatively high, is being inside previous discharges.
\end{abstract}

Keywords: Pockels, surface discharge, potential distribution, $\mathrm{SF}_{6}$, stepwise

\section{Introduction}

Surface discharge on a dielectric material has a great influence on the insulating performance of electrical apparatus and electronic devices. Information on the potential and the field profile along the surface discharge is required for quantitatively discussing and clarifying the propagation process.

Sensing techniques with a Pockels crystal have been applied to the space charge measurement of electrical discharge ${ }^{(1)(2)}$.

We proposed a new system based on sensing techniques with a Pockels crystal for measuring the transient potential distribution of surface discharge without any disturbance to the electric field distribution, and have been measuring mainly the potential distribution of a surface discharge in atmospheric air ${ }^{(5)-(9)}$. In this paper, the transient change of the potential distribution with a surface discharge in $\mathrm{SF}_{6}$ is measured with high space and time resolution.

\section{Measurement System}

Fig. 1 shows a schematic diagram of the measurement system used in this study. The system consists of an $\mathrm{Ar}$ ion laser, a cylindrical beam expander, a polarised beam splitter (PBS), $\mathrm{Bi}_{4} \mathrm{Ge}_{3} \mathrm{O}_{12}$ Pockels crystal (BGO, 16 in relative permittivity $\varepsilon_{r}$ ) with a diameter of $50 \mathrm{~mm}$, a streak camera and a CCD camera. As shown in Fig. 2, surface discharge occurs and propagates directly on the surface of the BGO.

Since the principle and the structure of this system have been already reported in the previous papers ${ }^{(6)-(8)}$, the details are omitted in this paper.

As the BGO crystal has the Pockels effect, the light intensity recorded with a streak and a CCD cameras, $I_{\text {out }}$ is given by

$$
I_{\text {out }}=\frac{I_{\text {in }}}{2}\left(1-\cos k V_{B G O}\right)(1)
$$

where $I_{\text {in }}$ is an input light intensity, $k$ is a constant and $V_{B G O}$ is the potential difference applied to the BGO.

To calibrate an exact potential value on the BGO, a plate electrode is attached on the insulating plate and the light intensities are measured for all points on the measuring surface of the plate while changing the voltage applied to the plate electrode. From these data, an experimental equation showing the relationship between the output light intensity and the potential on the BGO

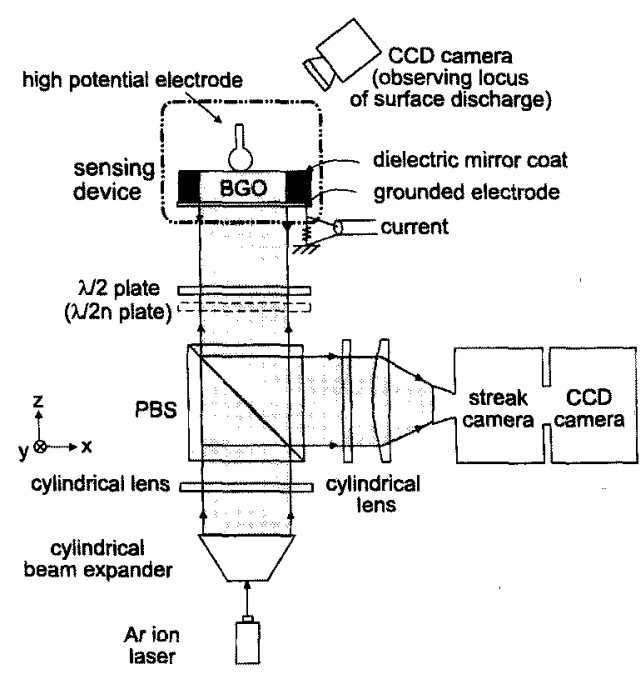

Fig. 1. Experimental setup. 


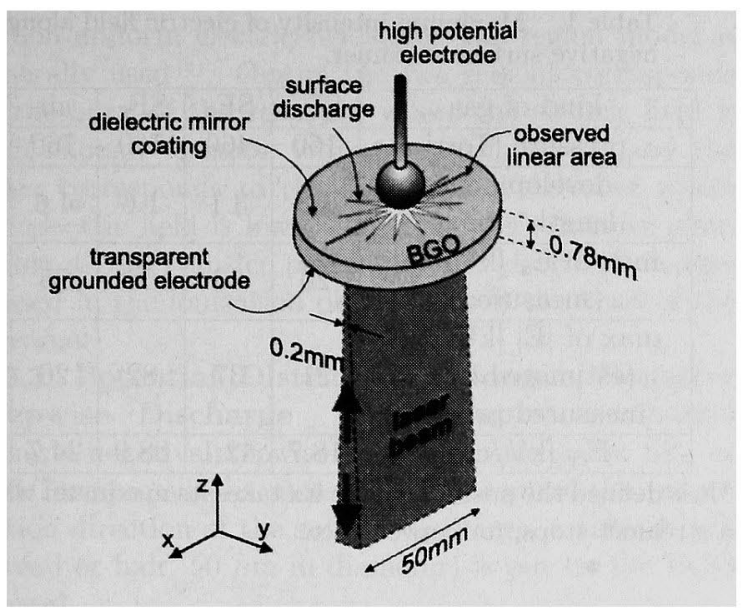

Fig. 2. BGO sensor device.

is derived for all 512 points along the measured linear path of $50 \mathrm{~mm}$.

With this system, the potential profile on the surface of the BGO along a linear path of $50 \mathrm{~mm}$ in maximum length can be measured quantitatively and continuously without causing any field disturbance. Considering the specification of the streak camera system, the minimum resolution of this system reaches $2-3$ ns in the domain and $0.05 \mathrm{~mm}$ in space domain ${ }^{(7)}$.

Equation (1) shows that $I_{\text {out }}$ changes as a cosine function of a potential difference applied to the BGO. This fact also suggests that there is a limited range in the measured potential difference for holding a single-value relationship between the detected light intensity and the potential difference. This limit is defined as a half wavelength voltage $V_{\pi}$. The value $V_{\pi}$ for this system is $14.5 \mathrm{kV}$. The minimum detectable potential depends on the stability of the laser output. Experimentally it is confirmed to reach $0.1 \mathrm{kV}^{(7)}$.

\section{Experimental Results}

3.1 Lichtenberg Figure Pattern of Surface Discharge Fig. 3 shows the Lichtenberg figure pattern of a negative surface discharge in $\mathrm{SF}_{6}$ at 380 Torr pressure. A $4 \mathrm{~mm} \phi$ sphere electrode is set on a $2 \mathrm{~mm}$ thickness PMMA plate and an impulse voltage of -44 $\mathrm{kV}$ in peak value is applied.

When a negative impulse voltage is applied to a sphere electrode set on an insulating material, negative surface discharge propagates radially at first from the electrode. This negative surface discharge is considered to consist of innumerable thin negative surface streamers ${ }^{(10)}$. If the applied voltage is increased, a surface leader is formed in the streamers and a stepwise subsequent discharge propagates in front of the previous discharge. When a thread or a scratch is set/cut on the insulating material, these stepwise subsequent discharges propagate along it. Fig. 3 corresponds to the Lichtenberg figure of such a stepwise discharge.

Fig. 4 shows the Lichtenberg figure pattern of a positive surface discharge under the similar experimental condition that of Fig. 3. An impulse voltage of 36

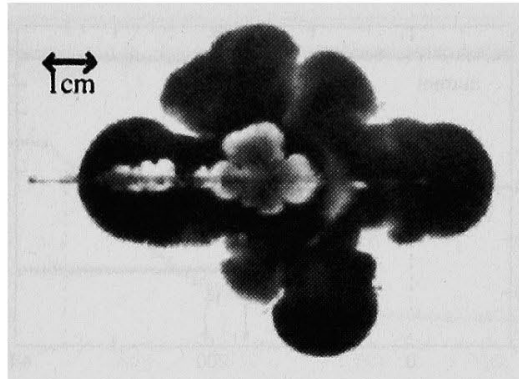

Fig. 3. Lichtenberg figure of a negative surface discharge in $\mathrm{SF}_{6}$ at 380 Torr pressure.

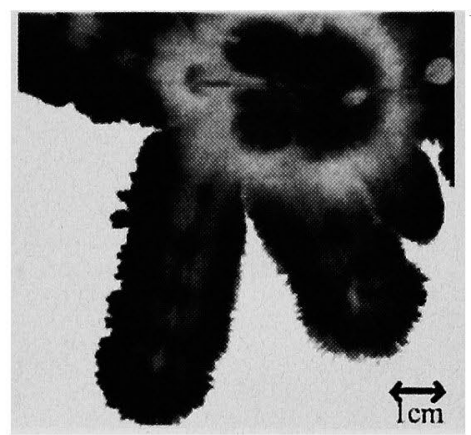

Fig. 4. Lichtenberg figure of a positive surface discharge in $\mathrm{SF}_{6}$ at 380 Torr pressure.

$\mathrm{kV}$ in peak value is applied to the $4 \mathrm{~mm} \phi$ sphere electrode. The positive surface discharge consists of many filament-like streamers. When the applied voltage is increased, a surface leader is also formed and stepwise subsequent discharges propagate in front of the previous discharge as well as the negative polarity.

In air or nitrogen, the positive surface discharge rarely propagates stepwise ${ }^{(11)}$, and it is hard to control the propagation direction of positive surface discharge. In $\mathrm{SF}_{6}$, stepwise subsequent discharge often propagates along a thread or a scratch set/cut on the insulating material.

The shape of the positive surface discharge is influenced considerably by the experimental condition. As the gas pressure is reduced, each filament-like positive streamer becomes thick and develops longer. As the intrinsic capacitance of the insulating plate is larger, the larger number of filament-like streamer occurs with a tight clearance and it becomes easier to control the propagation direction of surface discharge ${ }^{(11)}$.

3.2 Potential Distribution along a Negative Streamer A negative impulse voltage shown in Fig. 5 is applied to a $4 \mathrm{~mm} \phi$ sphere electrode that is set on a BGO crystal, and a negative surface discharge shown in Fig. 6 occurs from this electrode and develops on the $\mathrm{BGO}$ in $\mathrm{SF}_{6}$ at 160 Torr pressure. This negative surface discharge consists of innumerable thin negative surface streamers which develop radially from the electrode. The potential distribution along a negative surface streamer, which develops along the observed linear area, is measured. Fig. 7 shows the transient change in the potential $V(x, t)$ on the BGO surface with the prop- 


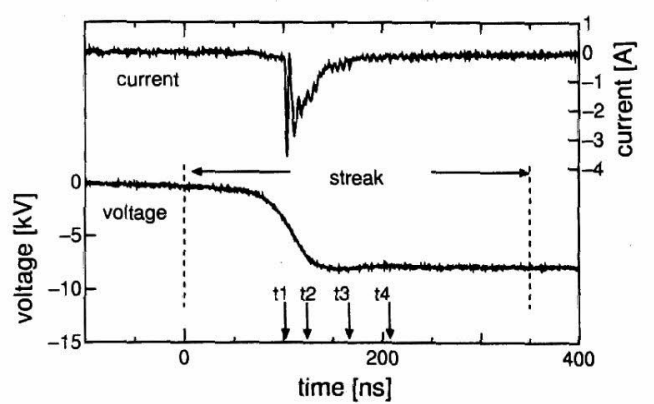

Fig. 5. Voltage and current profiles.

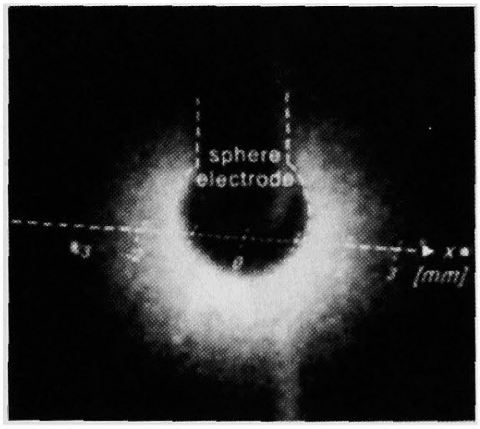

Fig. 6. Still photograph of negative surface discharge in $\mathrm{SF}_{6}$ at 160 Torr pressure.

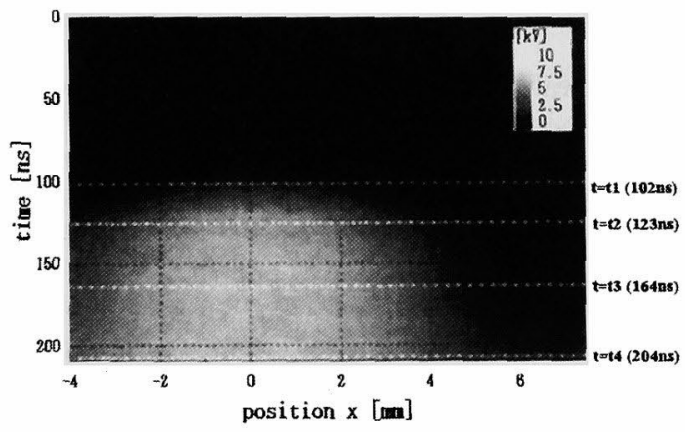

Fig. 7. Potential distribution of negative surface streamer in $\mathrm{SF}_{6}$ at 160 Torr pressure.

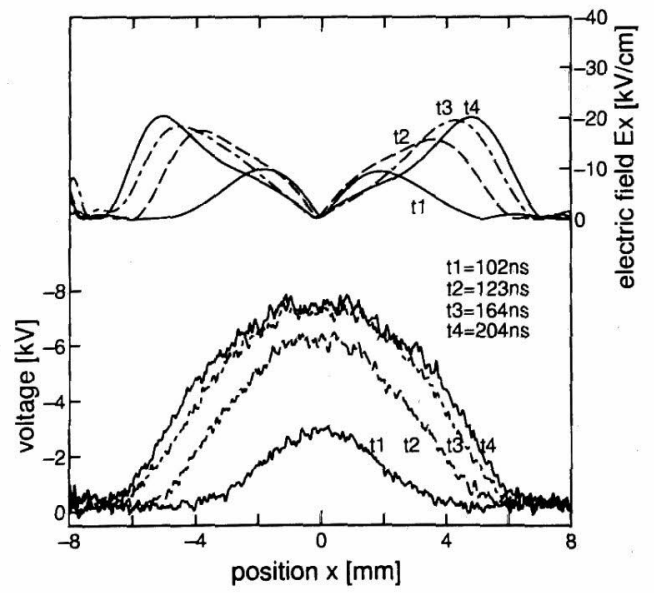

Fig. 8. Transient change in potential and electric field distribution along negative surface streamer in $\mathrm{SF}_{6}$ at 160 Torr pressure.
Table 1. Maximum intensity of electric field along negative surface streamer.

\begin{tabular}{|c|c|c|c|c|}
\hline kind of gas & $\mathrm{SF}_{6}$ & $\mathrm{SF}_{6}$ & $\mathrm{SF}_{6}$ & air \\
\hline pressure [Torr] & 160 & 460 & 760 & 760 \\
\hline $\begin{array}{c}\text { development } \\
\text { length* }{ }^{*}[\mathrm{~mm}]\end{array}$ & 4.8 & 3.1 & 1.6 & 4.6 \\
\hline $\begin{array}{c}\text { max of } \mathrm{E}_{\mathrm{x}}[\mathrm{kV} / \mathrm{cm}] \\
\text { (measured) }\end{array}$ & 20 & 33 & 58 & 25 \\
\hline $\begin{array}{c}\text { max of }|\mathrm{E}|[\mathrm{kV} / \mathrm{cm}] \\
\text { (estimated from } \\
\text { measured profile) }\end{array}$ & 21 & 37 & 82 & 26 \\
\hline $\mathrm{E}_{\mathrm{cr}}[\mathrm{kV} / \mathrm{cm}]$ & 18.7 & 42.1 & 88.9 & 24.7 \\
\hline
\end{tabular}

*It is defined the position where Ex takes its maximum when a streamer stops, for convenience.

agation of the surface streamer. The transient change in the potential distribution is shown in the bottom part of Fig. 8. These potential profiles are derived from Fig. 7 by extracting measured data on a line parallel to the t-axis.

The propagation direction components of the electric field $E_{x}$ can be calculated from the slope of measured potential distribution profiles. The potential profile is approximated with a polynomial function and $E_{x}$ profile is derived as its differential function.

To examine this fitting method, the potential and electric field distribution near a rod electrode $(2,5$ or $8 \mathrm{~mm}$ in diameter) were obtained and compared with the theoretical profile computed numerically using the charge simulation method. It was confirmed that the experimental result agreed with the theoretical one with $25 \%$ uncertainty in the maximum ${ }^{(12)}$.

The transient change in the distribution of $E_{x}$ is shown in the top part of Fig. 8. At each time, the distribution of $E_{x}$ has its maximum near the head of streamer. During the propagation of the surface streamer, the maximum value of $E_{x}$ varies around 19 $\mathrm{kV} / \mathrm{cm}$, which is the critical value of the electric field ionization in $\mathrm{SF}_{6}$ at 160 Torr pressure. The profiles of $|E|$ along the surface streamer can be calculated with charge simulation method from this measured potential profile ${ }^{(6)}$. In the neighbor of head of the streamer, the intensity of $|E|$ is estimated to excess $21 \mathrm{kV} / \mathrm{cm}$.

The potential distributions along a negative surface streamer in $\mathrm{SF}_{6}$ at 460,760 Torr pressure are also measured with the same method.

The maximum values of $E_{x}$ and $|E|$ at each pressure are shown in Table 1 , in comparison with the critical electric field strength $E_{c r}$ for the gaseous ionization, These data are measured under applying impulse voltage of $7 \sim 10 \mathrm{kV}$ in peak value to the electrode. It is possible to say $|E|$ near the head of streamer in $\mathrm{SF}_{6}$ keeps its value around electric-field ionization intensity.

The value of $E_{x}$ in the stem of a streamer in $\mathrm{SF}_{6}$ decreases gradually with the propagation of the streamer. At $x=3 \mathrm{~mm}$ it decreases from $15.0 \mathrm{kV} / \mathrm{cm}(t=\mathrm{t} 2)$ to $12.6 \mathrm{kV} / \mathrm{cm}(t=\mathrm{t} 4)$. While a streamer propagates, the conductivity in the stem of it is being increased.

For the physical explanation of the surface discharge 
in non-uniform electric fields, the two-region model is generally used (3). One of the two regions corresponds to the head of the streamer where the electric field is high enough to cause ionization phenomena, and the other corresponds to the stem of the streamer where the electric field is low. The stem of a streamer plays a role as the transfer passage of the electrical charges caused in the ionization phenomena at the head of the streamer.

3.3 Potential Distribution along a Negative Stepwise Discharge The potential distribution along a negative stepwise surface discharges in $\mathrm{SF}_{6}$ at 760 Torr pressure is measured. To control the propagation direction of the surface discharge, a thread (silk thread or hair, $50 \mu \mathrm{m}$ in diameter) is put on the BGO crystal.

Figs. 9, 10 and 11 show applied voltage waveform, the transient change in the potential $V(x, t)$ on the BGO and potential and $E_{x}$ distributions along it, respectively. A $6 \mathrm{~mm} \phi$ circular cone electrode is used. The divergence angle of its tip is 30 degree. The conical electrode is set just on the position of $x=0 \mathrm{~mm}$, and the impulse voltage is stressed at $t=-350 \mathrm{~ns}$. The streak camera observing the potential distribution starts at $t=0 \mathrm{~ns}$ and ends at 348 ns.

The first surface discharge (stage "I") occurs around $t=-200 \mathrm{~ns}$ and the surface discharge propagates forming two more steps in the direction of the right (stages "II" and "III") as shown in Fig. 12. Simultaneously with these step propagations, current pulses appear in Fig. 9 and the high potential region expands in Fig. 10.

Since there is little change between the potential distribution profiles at $t=\mathrm{t} 5 \& \mathrm{t} 6$, and $t=\mathrm{t} 9 \& \mathrm{t} 10$, it is natural to say the discharge at the stage "III" starts around $t=140 \mathrm{~ns}$ and stops around $t=180 \mathrm{~ns}$.

Considering $E_{x}$ profiles at t 6 shifted the streamer development length (equals to $0.7 \mathrm{~mm}$ ) along the $\mathrm{x}$-axis, it coincides well with that of t10 around the head of the discharge. As well as stepwise negative surface discharge in the atmospheric air ${ }^{(7)}$, it can be said that the $E_{x}$ distribution around the head of the surface discharge keeps a particular profile when the surface discharge stops.

During the propagation of the discharge at stage "III", a leader is being formed inside previous discharges. For example, the value of $E_{x}$ at $x=1.3 \mathrm{~mm}$ decreases from $40.2 \mathrm{kV} / \mathrm{cm}$ (at $t=\mathrm{t} 6$ ) down to less than $17 \mathrm{kV} / \mathrm{cm}$ (at $t=\mathrm{t} 7$ ). At the time $t=\mathrm{t} 7$, the value of $E_{x}$ in the region of $x=0 \sim 1.5 \mathrm{~mm}$ is below $10 \mathrm{kV} / \mathrm{cm}$.

3.4 Potential Distribution along a Positive Streamer The potential distribution along a positive surface streamer in $\mathrm{SF}_{6}$ at 160 Torr pressure is measured with the same method as mentioned in the section 3.2. The experimental conditions are set similar to those of the section 3.2 except for the polarity.

Figs. 13, 14 and 15 show the applied voltage waveform, the still photograph of a positive surface discharge and potential and $E_{x}$ distributions along it, respectively.

Compared with the negative surface streamer described in section 3.1, there are small differences in the

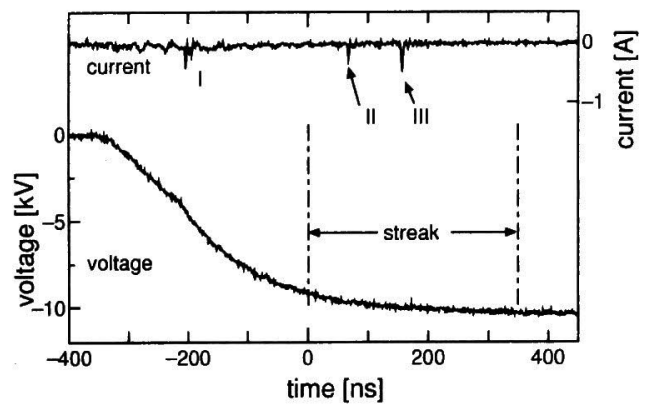

Fig. 9. Voltage and current profiles.

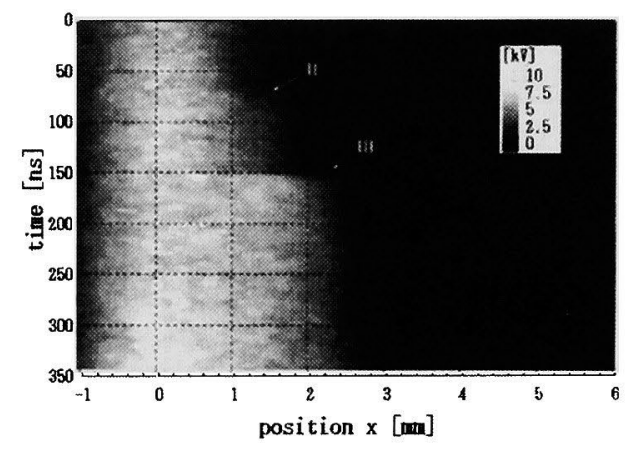

Fig. 10. Potential distribution of negative stepwise surface discharge in $\mathrm{SF}_{6}$ at 760 Torr pressure.

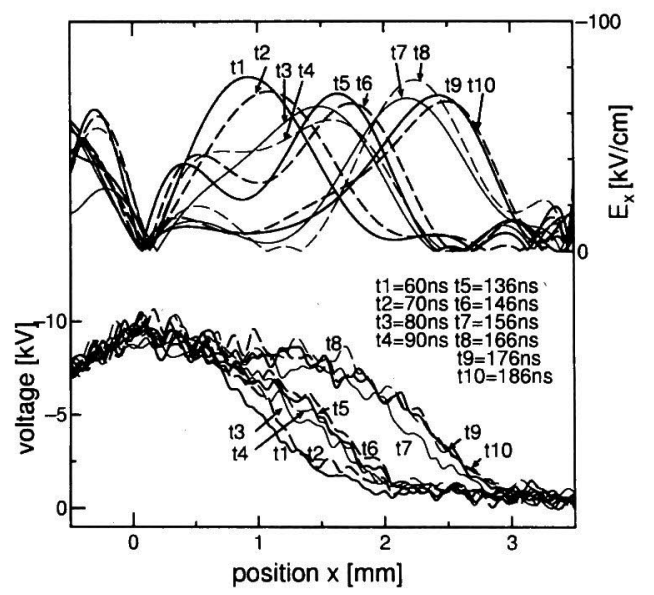

Fig. 11. Transient change in potential and electric field distribution along negative stepwise surface discharge in $\mathrm{SF}_{6}$ at 760 Torr pressure.

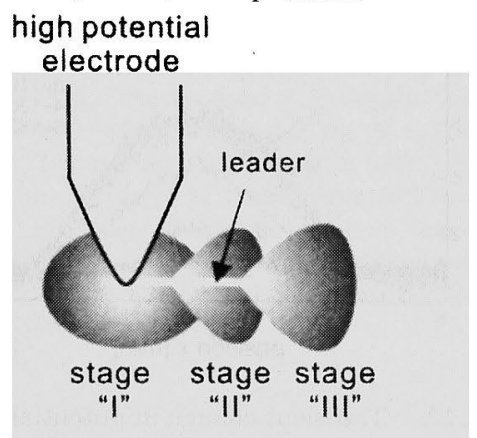

Fig. 12. Schematic illustration of negative stepwise discharge. 
shape and the intensity of $E_{x}$ distribution.

As the gas pressure is reduced to 160 Torr, each filamentous streamer becomes thick. Furthermore, as the intrinsic capacitance of an insulating plate is relatively large compared with that of $2 \mathrm{~mm}$ thickness PMMA plate, larger number of streamer occur densely. From the still photograph, it is difficult to recognize each filament-like streamer.

At any time, the distribution of $E_{x}$ has a triangular profile and $E_{x}$ has its maximum near the head of the positive streamer. The value of $E_{x}$ around the positive streamer head keeps around the critical value for the gas ionization $(18.7 \mathrm{kV} / \mathrm{cm})$.

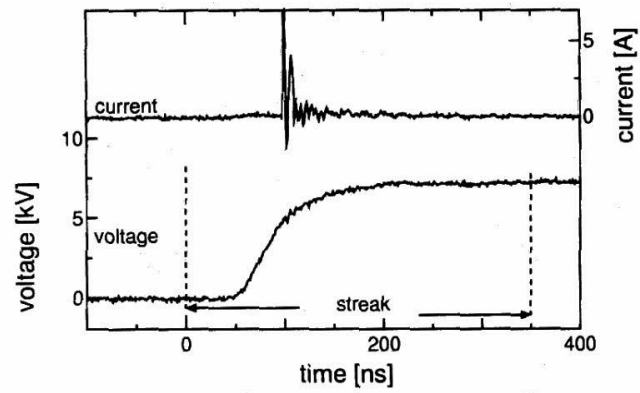

Fig. 13. Voltage and current profiles.

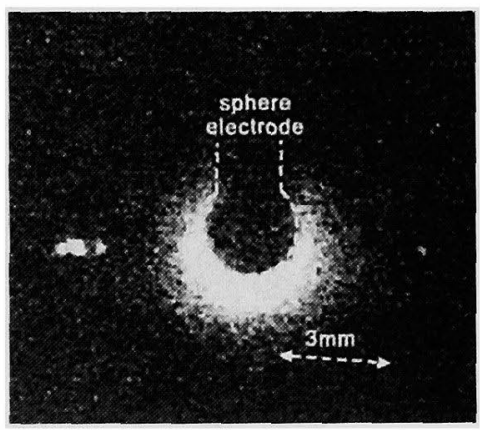

Fig. 14. Still photograph of positive surface discharge in $\mathrm{SF}_{6}$ at 160 Torr pressure.

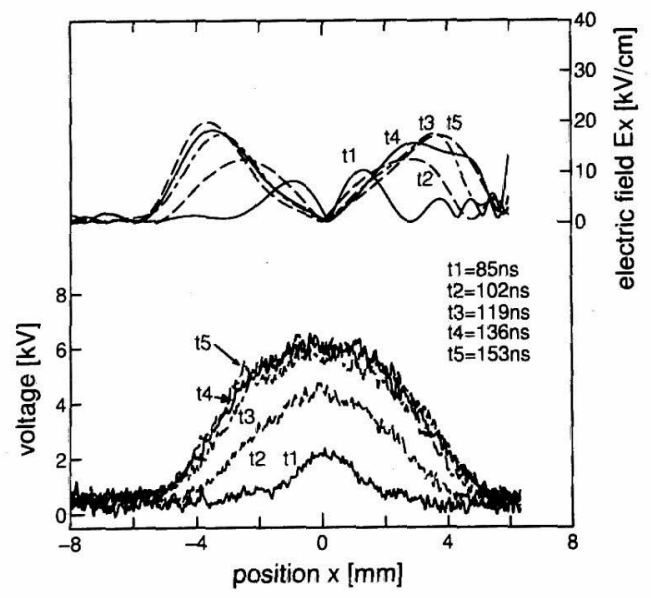

Fig. 15. Transient change in potential and electric field distribution along positive surface streamer in $\mathrm{SF}_{6}$ at 160 Torr pressure.
This is in contrast to a positive streamer developing in atmospheric air ${ }^{(8)}{ }^{(9)}$. In case of the positive streamer in air, the potential decreases linearly with increasing the length $x$ except in the neighbor of the electrode and $E_{x}$ in the streamer is kept a low electric field less than the critical value for the gas ionization.

This difference is due to the shape of discharge. The filament of positive streamers in the atmospheric air has a very sharp tip shape, and propagates keeping a distance each other while that in $\mathrm{SF}_{6}$ at 160 Torr pressure propagates densely. In the case of the atmospheric air, the electric field component perpendicular to the propagating direction is high and totally the electric field strength $|E|$ can excess the critical value for the gas ionization.

3.5 Potential Distribution along a Positive Stepwise Discharge The potential distribution along a stepwise positive surface discharges in $\mathrm{SF}_{6}$ at 360 Torr pressure is measured. Figs. 16, 17, 18 and 19 show the applied voltage waveform, the still photograph of a positive stepwise surface discharge, the transient change in the potential $V(x, t)$ on the BGO and potential and $E_{x x}$ distributions along it, respectively.

The first surface discharge (stage "I") occurs around $t=-250 \mathrm{~ns}$ and the surface discharge propagates having two more steps in the direction of the left as shown in Fig. 18. The second discharge (stage "II") starts to propagate at $t=\mathrm{t} 1$ and stops at $t=\mathrm{t} 4$. The third discharge (stage "III") starts at $t=\mathrm{t} 5$ and stops at $t=$ t10. $E_{x}$ reaches its maximum at $x=-3.7 \mathrm{~mm}(t=\mathrm{t} 1)$, $x=5.4 \mathrm{~mm}(t=\mathrm{t} 5)$ and $x=-8.0 \mathrm{~mm}(t=\mathrm{t} 10)$, respectively. From Fig. 17, it is difficult to distinguish the second discharge from the third discharge. The $E_{x}$ distribution around the streamer head of third discharge $(t=\mathrm{t} 10)$ is similar in profile to that of the first discharge

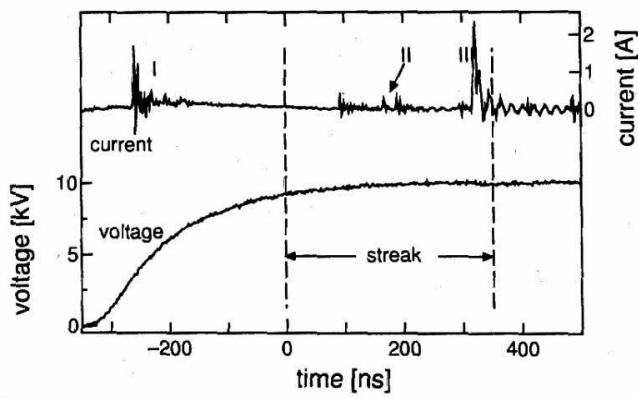

Fig. 16. Voltage and current profiles.

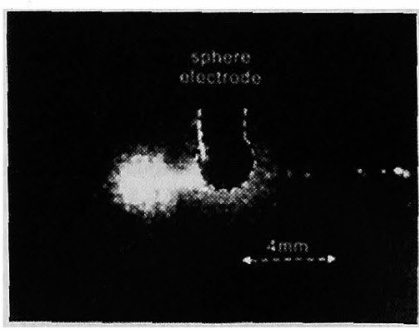

Fig. 17. Still photograph of stepwise positive surface discharge in $\mathrm{SF}_{6}$ at 360 Torr pressure. 


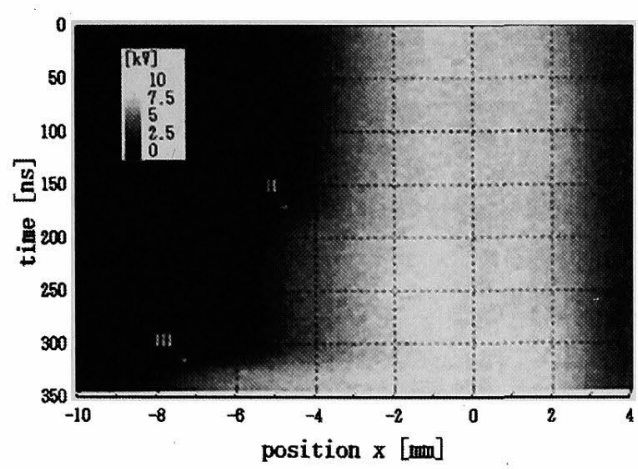

Fig. 18. Potential distribution of stepwise positive surface discharge in $\mathrm{SF}_{6}$ at 360 Torr pressure.

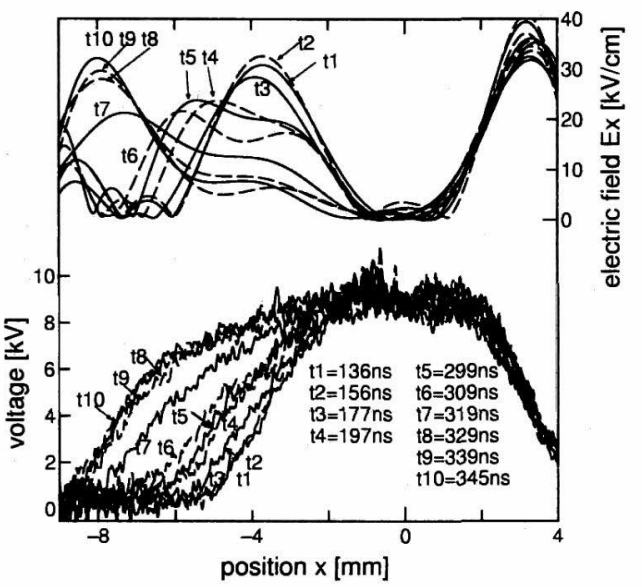

Fig. 19. Transient change in potential and electric field distribution along stepwise positive surface discharge in $\mathrm{SF}_{6}$ at 360 Torr pressure.

$(t=\mathrm{t} 2)$. As well as the negative polarity, the $E_{x}$ distribution around the head of the surface discharge keeps a particular profile in principle when the surface discharge stops.

With the propagation of these subsequent discharges, the leader is formed inside previous discharges just like the negative stepwise discharge described in the section 3.2. At the time $t=\mathrm{t} 8$, the value of $E_{x}$ in the region of $x=0 \sim 5.4 \mathrm{~mm}$ is below $7 \mathrm{kV} / \mathrm{cm}$.

\section{Conclusion}

An optical method for measuring the transient potential distribution of surface discharge using Pockels effect was developed. The potential distributions along a surface discharge in $\mathrm{SF}_{6}$ at $160 \sim 760$ Torr are measured with high time and spatio resolution.

The experimental results obtained from the measurement are summarized as follows:

(1) The distribution of $E_{x}$ along a negative streamer has a triangular profiles and $E_{x}$ has its maximum near the streamer head. The value of $E_{x}$ around the streamer head keeps around the critical value requiring for the gas ionization.

(2) In contrast to the potential distribution along a streamer in air, little significant difference is recognized between the potential distribution along a positive streamer in $\mathrm{SF}_{6}$ and that of a negative one. The potential distribution profile along a streamer is sensitive to the shape and arrangement of the filament-like streamers.

(3) During the propagation of the subsequent stepwise discharge, the leader is being formed inside previous discharges. The electric field intensity $E_{x}$ inside the negative leader at 760 Torr pressure is less than $10 \mathrm{kV} / \mathrm{cm}$. That inside the positive leader at 360 Torr pressure is less than $7 \mathrm{kV} / \mathrm{cm}$.

(Manuscript received December 25, 2000, revised March 15, 2001)

\section{References}

(1) K.Hidaka, IEEE Electrical Insulation Magazine 12 17, 1996

(2) T.Kawasaki et al., J. Appl. Phys. 76 3724-3729, 1994

(3) I. Gallimberti, J. Phys. D: Appl. Phys. 5 2179-2189, 1972

(4) S.Larigaldie, J. Appl. Phys. 61 90-108, 1987

(5) A.Kumada, M.Chiba and K.Hidaka, J. Appl. Phys., 84 3059-3065, 1998

(6) A.Kumada, M.Chiba and K.Hidaka, Trans. IEE Japan 118 A $723-728,1998$ (in Japanese)

(7) A.Kumada, M.Chiba and K.Hidaka, Trans. IEE Japan 120B $75-80,2000$

(8) A.Kumada, M.Chiba and K.Hidaka, Trans. IEE Japan 120A $204-210,2000$ (in Japanese)

(9) A.Kumada et al., Annual Report Conference on Electrical Insulation and Dielectric Phenomena (CEIDP/IEEE), Vol.1, pp. 150-153, Austin, 1999

(10) M.Sone et al., Trans. IEE Japan 94-A 523-529, 1974 (in Japanese)

(11) M.Chiba et al., Trans. IEE Japan 108-A 162-168, 1988 (in Japanese)

(12) A.Kumada, Ph.D Thesis, The University of Tokyo, December, 1998 (in Japanese)

Akiko Kumada (Member) She was born on 9 February,

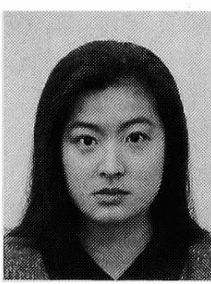
1972. She received the B.S., M.S. and Dr. degrees in electrical engineering from the University of Tokyo in 1994, 1996 and 1999, respectively. Since 1999 she has been at the department of advanced energy, graduate school of frontier sciences, in the same university as a research associate. Her research interests include optical high voltage measurement and propagation mechanism of surface discharges. Her work won a paper award from IEE of Japan in 2000.

Masakuni Chiba (Member) He was born on 8 October,

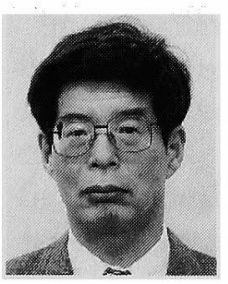
1942. He received B.S. degree from Nihon University in 1966 and the M.S. degree from Tokyo Denki University in 1970. Since 1966 he has been at Department of Electrical Engineering of the University of Tokyo as a research associate. He has been engaged in the studies of high voltage techniques and electrical discharges. 
Kunihiko Hidaka (Member) $\mathrm{He}$ was born on 27 April, 1953. He received B.S., M.S. and Dr. degree
in electrical engineering from the University of Tokyo in 1976, 1978 and 1981, respectively. $\mathrm{He}$ is a professor at the Department of Electrical Engineering of the University of Tokyo. He has been engaged in the research of electrical breakdown phenomena concerned with

high voltage technology. His work won premiums and paper awards from both British IEE and IEE of Japan 